\title{
Estado actual de la hipertensión pulmonar en 3 países de Latinoamérica y la Sociedad Latina de Hipertensión Pulmonar
}

\section{Important growth in Latin America concerning approach, diagnosis, and treatment of pulmonary arterial hypertension}

Jaime Eduardo Morales-Blanhir(1); María de Jesús Rosas Romero(2); Julio Robledo Pascual(3); Alejandro Londoño V.(4);

Douglas Olivares Barroeta ${ }^{(5)}$; Migdalia Denis( ${ }^{(6)}$

\section{RESUMEN}

Importante crecimiento en Latinoamérica sobre abordaje, diagnóstico y tratamiento de la Hipertensión Pulmonar.

En México, los pacientes acuden en estadios avanzados, la propuesta de trabajo es: aumentar centros de referencia, tener disponibilidad de nuevos medicamentos, extender enseñanza a médicos y pacientes, y establecer el Consenso de diagnóstico y tratamiento.

En Colombia el Comité Técnico Científico lleva a cabo estrategias para realizar diagnósticos en fases tempranas de la enfermedad, educación continua y centros de referencia para distintas labores. Otra idea de los grupos de trabajo es que el paciente porte un resumen electrónico (USB), en caso de atención médica de urgencias.

En Venezuela se propone como meta: obtención de medicamentos, mayor información a los pacientes, orientar sobre el apoyo psicológico, ayudar a los pacientes con gastos.

Las Sociedades de Hipertensión Pulmonar para Pacientes en Latinoamérica, ofrecen impulso a las instituciones públicas y privadas por lo que se debe tomar como ejemplo para el desarrollo y expansión a otros países, permitiendo un cambio en el panorama en que viven los pacientes con hipertensión pulmonar en toda Latinoamérica.

Palabras clave:hipertensión pulmonar, grupos de trabajo, organizaciones no gubernamentales.

\section{ABSTRACT}

In Mexico, patients seek help when the disease is advanced. The working proposal is: to increase the number of referral centers, to increase the availability of new drugs, to improve physician and patient education, and to establish the Diagnosis and Treatment Consensus.

(1) Internista, Neumólogo. Instituto Nacional de Ciencias Médicas y Nutrición Salvador Zubirán, Ciudad de México. Director del Departamento de Circulación Pulmonar, Asociación Latinoamericana de Tórax (ALAT). Director Médico de la SociedadLatina de Hipertensión Pulmonar (SLHP). México.

(2) Q.F.B. Investigador Asociado A, Instituto Nacional de Ciencias Médicas y Nutrición Salvador Zubirán, Ciudad de México. Correo electrónico: reirosasr@yahoo.com.mx

(3) Intensivista, Neumólogo. Hospital Regional de Alta Especialidad Dr. Juan Graham Casasús, Villahermosa Tabasco. Correo electrónico: jrobledopascual@yahoo.com

(4) Internista Neumólogo. Clínica Cardiovascular, Medellín, Colombia. Correo electrónico: alv@une.net.co

(5) Neumólogo. Hospital de Clínicas Caracas, Venezuela. Correo electrónico: olivaresdouglas@gmail.com

(6) Presidente, Sociedad Latina de Hipertensión Pulmonar. Estados Unidos. Correo electrónico: phlatinsociety@hotmail.com

Correspondencia: Dr. Jaime Eduardo Morales Blanhir, E-mail: moralesjaime@usa.net

Recibido: febrero 10 de 2011. Aceptado: febrero 20 de 2011. 
In Colombia, the Scientific-Technical Committee is developing strategies to diagnose the disease in its early stages, to develop continuing education, and to increase the number of referral centers for different duties. Another idea of the working parties is to encourage patients to carry with them an electronic summary (USB), in case of emergency medical attention.

In Venezuela, the proposed goals were the following: improved access to drugs, more information to patients, psychological support, helping patients with the expenses.

In Latin America, Societies of Patients with Pulmonary Hypertension offer impulse to public and private institutions. They should be regarded as an example for development and expansion in other countries, which would have a positive impact on the conditions of life of patients with pulmonary arterial hypertension in Latin America.

Key words: pulmonary arterial hypertension, working parties, non-government organizations

Rev Colomb Neumol 2011; 23(1): 16-21.

En lo últimos años, se ha tenido un importante crecimiento en Latinoamérica (LA) sobre el abordaje, diagnóstico y tratamiento de la hipertensión pulmonar. De esta manera, vemos los avances a nivel médico en Brasil, Argentina, Colombia, Venezuela, Chile, Perú, Bolivia, Paraguay, Uruguay, República Dominicana y México. En el pasado agosto 2009 en la ciudad de Río de Janeiro Brasil, se mostraron los avances en varios países al respecto, como es el caso de México, Colombia y Venezuela que se describen.

La organización de las diferentes Sociedades de Pacientes con Hipertensión Pulmonar de Argentina, Brasil, Colombia, España, México, Perú, Puerto Rico, Venezuela, República Dominicana y EE.UU, han ido creciendo en conjunto con la Sociedad Latina de Hipertensión Pulmonar (SLHP), mostramos un aspecto general de las mismas

\section{SITUACIÓN ACTUAL EN MÉXICO}

México es un país con una población de 103263388 habitantes, el $49 \%$ son hombres y $51 \%$ mujeres, con una edad promedio de 24 años, el $60 \%$ de la población se encuentra entre los 15 -59 años. La edad mediana de la maternidad es de 24 años. El 94\% asiste a la escuela de los 6 a los 14 años. La esperanza de vida está en 75 años. La población con servicios de salud es $47 \%$, al IMSS $31 \%$, al ISSSTE $6 \%$ y al seguro popular $7 \%$.

Para conocer la situación actual en México de la Hipertensión Pulmonar (HP), se realizó una encuesta vía electrónica, a 300 médicos Neumólogos, 300 Internistas, 300 de Terapia Intensiva. Se formularon 5 preguntas básicas: ¿Cómo realiza el diagnóstico de hipertensión pulmonar?, ¿Qué tratamientos utiliza para la hipertensión pulmonar?, ¿Conoce las fundaciones de pacientes con hipertensión pulmonar en México? , ¿Qué le gustaría recibir para la educación médica continua de médicos y pacientes de este tema al respecto, en su Estado?, ¿Cómo ve las perspectivas futuras de la hipertensión pulmonar en México?. El análisis de las respuestas es el siguiente:

Los pacientes con hipertensión pulmonar acuden a la atención médica en estadios avanzados de la enfermedad. El diagnóstico se realiza la mayoría de las veces por ecocardiograma y existen pocos centros que realizan cateterismos cardiacos derechos. Se cuenta con el tratamiento convencional, bloqueadores de los canales de calcio y con la disponibilidad de los nuevos medicamentos como iloprost que llegó a México 2007, bosentán en el 2008, treprostinil y sildenafilo en el 2010; el de mayor uso es el sildenafilo. En el tratamiento quirúrgico se cuenta con la septostomía, con una amplia experiencia; la tromboendarterectomía se realiza en dos centros de alta especialidad uno de ellos inició en el 1994 y el trasplante pulmonar con realización limitada. Al momento tenemos pocos centros de referencia especializados para la atención de la hipertensión pulmonar.

Las debilidades en las que se debe trabajar en México son: aumentar el número de centros de referencia, favorecer la disponibilidad a los pacientes de los nuevos medicamentos, hacer extensa la enseñanza de la HP a los médicos y los pacientes. Establecer el Consenso de diagnóstico y tratamiento aplicado a nuestro medio.

Actualmente se están realizando esfuerzos en la educación médica continua, con la enseñanza a médicos Internistas, Reumatólogos, Cardiólogos y Neumólogos sobre el diagnóstico y los tratamientos actuales de la HP. Se publicó el Manual de Hipertensión 
Pulmonar para Pacientes, con una difusión a los mismos de las diferentes Sociedades de HP de LA, actualmente en su segunda edición, cabe mencionar que es el primer material realizado en su forma original en español. Se trabaja en conjunto con las Sociedades Mexicana de Neumología y Cardiología, con la primera se están realizando también cursos de actualización. Con el Departamento de Circulación Pulmonar de la ALAT se está trabajando en conjunto en el Registro y el Consenso Latino de HP. El Primer Curso Itinerante de HP, ALATSMNYCT se realizó durante el Congreso de la Sociedad Mexicana de Neumología 2010. Se está trabajando con la SLHP con Migdalia Denis Presidente, estableciéndose redes de pacientes y médicos para el diagnóstico y tratamiento de la HP.

\section{SITUACIÓN ACTUAL EN COLOMBIA}

Como en varios países del continente y del mundo, en Colombia se ha despertado el interés por la Hipertensión Arterial Pulmonar (HAP) esto es debido a que se puede modificar la historia natural de una enfermedad que hace unos años era fatal.

El ofrecer una nueva esperanza de mejor calidad y cantidad de vida a pacientes con HAP y a sus familias hacen que el reto del enfoque del manejo de la enfermedad sea una prioridad. La consolidación paulatina de centros de referencia para el manejo integral de la HAP y el interés y fortalecimiento por parte de la Asociación Colombiana de Neumología y Cirugía de Tórax (Asoneumocito), y de su departamento de HAP y Circulación Pulmonar, mediante un trabajo ordenado y planeado, en poco tiempo ha mostrado logros importantes.

La mayor cobertura en salud, del sistema de la seguridad social a todos los colombianos es importante para mejorar el acceso de pacientes con HAP a una atención adecuada. En su momento, la figura de la "Tutela", donde el ciudadano reclama, amparado en la constitución colombiana, un derecho propio a la salud, y más reciente con la figura del Comité Técnico Científico (CTC), mecanismo orientado a aprobar y pagar, por parte del asegurador en salud, determinados procedimientos diagnósticos y los tratamientos requeridos por el médico tratante. Estas estrategias permiten realizar un buen diagnóstico en fases más tempranas de la enfermedad, con resultados satisfactorios.

Con respecto al diagnóstico y exámenes específicos, utilizados en el estudio y seguimiento de la HAP tenemos los biomarcadores (BNP, ProBNP, Troponina), encontramos que su medición para establecer riesgo, pronóstico y herramienta para el seguimiento, no es una práctica extendida en todo el país y solo unos pocos sitios lo hacen de rutina en la actualidad.
Las otras pruebas de diagnóstico realizadas: AngioTAC de arterias pulmonares, ecocardiografía, gamagrafía $\mathrm{V} / \mathrm{Q}$, resonancia magnética. Esta última, con buenos resultados en la valoración de la función ventricular derecha, sobretodo en los pacientes candidatos a trasplante pulmonar. El cateterismo cardiaco derecho para confirmar el diagnóstico y para evaluar la reactividad vascular pulmonar es un procedimiento que cada vez se hace con mayor frecuencia, aunque la técnica no está estandarizada en todos los centros e indistintamente se usa óxido nítrico, adenosina e iloprost.

En cuanto al tratamiento, contamos con la mayoría de fármacos para el manejo adecuado de la HAP, todos ellos aprobados por el INVIMA (ente regulador en Colombia de la aprobación de los fármacos): Sildenafil (Viagra $\left.{ }^{\circledR}\right)$, Bosentan (Tracleer®), Iloprost (Ventavis®) y recientemente Ambrisentan (Volibris $\AA$ ) y Epoprostenol ( Flolan®).

Existen además en el país, algunos centros especializados que hacen tromboendarterectomía pulmonar indicada en la hipertensión pulmonar tromboembólica y atrioseptostomía, como terapia puente al trasplante pulmonar bilateral o al trasplante en bloque de corazón y pulmón en la Clínica Cardiovascular, Medellín, Colombia.

En cuestiones de educación médica continuada se han adelantado algunas actividades en distintas ciudades del país con la colaboración de Asoneumocito y su comité de HAP, y participa en distintas reuniones del Departamento de Circulación Pulmonar de la ALAT.

A nivel del comité de HAP de Asoneumocito se trabajó en la elaboración de las guías de manejo adaptadas a nuestro medio, las cuales facilitarán el entendimiento de la enfermedad, su diagnóstico precoz y su adecuado tratamiento. Estamos seguros de que se convertirán en corto plazo en una valiosa herramienta de consulta y de manejo de la HAP en el país y facilitará los trámites ante las aseguradoras para que los pacientes tengan medicación con la oportunidad que se precisa.

En los distintos centros de referencia se vienen planeando protocolos de investigación y tenemos la idea de que sean compartidos entre ellos, de tal manera que la casuística sea significativa. Uno de estos proyectos es una encuesta multicéntrica, (HIAPULCO) orientada a conocer datos demográficos, prevalencia de la enfermedad, como se hace el diagnóstico, como se tratan los pacientes y como se hace su seguimiento. Además estamos interesados en la puesta en marcha del Registro Latino de HP (RELAHP), actualmente en desarrollo, donde representantes de Colombia han invertido un tiempo significativo, en especial de uno de los miembros del comité y de un ingeniero de sistemas contratado para la elaboración de la base de datos. 
Con respecto a programas dirigidos a pacientes tenemos que decir que la Fundación Colombiana de Hipertensión Pulmonar®, ha logrado nuevas afiliaciones y convenios que facilitarán la rehabilitación integral, ocupacional y social del paciente con la enfermedad. A través de esta fundación de pacientes se estudia la posibilidad de que cada uno porte un carnet que lo identifique como paciente y esto le daría derecho a algunas facilidades, por ejemplo, estudio o trasporte público.

Otra idea que ha surgido de los grupos de trabajo es que cada paciente porte un resumen electrónico de su propia historia (en una memoria USB), de tal manera que si requiere atención médica de urgencias, el médico tratante de turno, pueda orientar la consulta como es debido.

A futuro se prevé algunos puntos a desarrollar en el comité de HAP de Asoneumocito y de ALAT, que ayuden al progreso del manejo de la misma, ellos son: la consolidación del RELAHP, depurar el correcto diagnóstico y clasificación de la HAP, tratar de fomentar la creación de clínicas especializadas en el diagnóstico y manejo de HAP y difundir e implementar en las diferentes regiones del país, las nuevas guías colombianas de manejo de la HAP.

Por último, nos resta mencionar que nos preocupan los altos costos de los medicamentos y la carga de la enfermedad en términos de años de vida saludable perdidos. Por lo anterior, estamos trabajando en el diseño de un protocolo que nos permita averiguar la relación de costo y de beneficio, porque somos responsables del gasto en salud, en un porcentaje importante, en un país que tiene dificultades presupuéstales. Los resultados de dicho estudio ayudarán a buscar la manera para disminuir costos y eso hará que más colombianos tengan mayor acceso a los servicios de salud.

En Medellín, Colombia, se llevó acabo el II Encuentro Colombiano de HAP, los días 5 y 6 de marzo 2010. Fue una actividad académica de muy alto nivel científico, provechosa para la formación profesional de los asistentes. En este mismo evento se presentaron las Guías para el Diagnóstico y Tratamiento de la Hipertensión Pulmonar. Avalado por la Asociación Colombiana de Neumología y Cirugía de tórax, Sociedad Colombiana de Cardiología y Cirugía Cardiovascular, Asociación Colombiana de Reumatología y la Asociación Colombiana de Infectología.

\section{SITUACIÓN ACTUAL EN VENEZUELA}

La Fundación Venezolana de Hipertensión Pulmonar (FUNDAVHIP) es una organización privada sin fines de lucro, conformada por las siguientes personas:
Presidente: Anabel Sivira

Vicepresidente: Dr. Rafael Cabrera

Gerente General: Dr.Santiago Guzmán

Director tecnico: Dr.Douglas Olivares

Vocal: Dra. Angela de Martínez

Vocal: Maria Eugenia Sosaya

Es una respuesta al poco conocimiento de los tratamientos que pueden ser aplicados a la enfermedad, al escaso material de apoyo que se tiene en nuestro país sobre la hipertensión pulmonar (HP) conllevando esta situación a una serie de casos mal y otros tardíamente diagnosticados, y sin el tratamiento adecuado. Además de atender la necesidad de establecer una vía expedita y segura para la obtención de medicinas y para tratar esta enfermedad en sus diferentes etapas.

Lo objetivos inmediatos que, nos propusimos acometer son los siguientes: a) Obtención de medicamentos para el tratamiento de la enfermedad. b) Proveer de información a las personas afectadas con HP. Orientar sobre la asistencia medica psicológica, así como ayudar a los pacientes a sufragar algunos de sus gastos.

Hasta la fecha hemos obtenido los siguientes logros: La creación y consolidación de FUNDAVHIP como la única Institución privada que trabaja coordinadamente con el Estado para auxiliar a los pacientes con esta enfermedad. De tal forma que se ha sensibilizado a los diferentes entes de salud gubernamentales sobre la HP, realizando múltiples intervenciones ante la Asamblea Nacional, Ministerio de Sanidad para el Poder Popular, Instituto Nacional de Higiene, Instituto Venezolano de los Seguros Sociales y la Industria Farmacéutica, consiguiendo el apoyo de la Sociedad Venezolana de Neumología y de la Sociedad Venezolana de Cardiología. Se han creado capítulos de la Fundación en la capital así como en el interior del país como son: Fundacardin en el Hospital Militar Carlos Arvelo, Ascardio en Barquisimeto Estado Lara, y en el Hospital Universitario de Maracaibo en el Estado Zulia.

Realización de Simposios, Jornadas y Talleres de HP, auspiciados por las Sociedades de Cardiología y Neumología, y de una publicación especial en la revista de la Sociedad Venezolana de Cardiología.

Luego de múltiples reuniones y conversaciones con el Estado se logro la formación del programa de hipertensión pulmonar IVSS único en Latinoamérica, simultáneamente se estableció el programa de HP para el personal Militar IPSFA. Se ha estimulado a la Industria Farmacéutica a fin de capacitar cada día a los médicos comprometidos con esta patología. 
Mediante múltiples intervenciones en medios audiovisuales FUNDAVHIP tiene activa presencia en medios de comunicación en el país. Ha también establecido lazos con la Asociación Española de HP.

En Agosto del 2009 se realizó en el Hospital de Clínicas Caracas el primer encuentro nacional de Pacientes con HP, habiendo sido exitosa dicha reunión, con asistencia importante de pacientes con la enfermedad.

Tenemos retos importantes en el futuro, siendo necesaria la apertura de Unidades de apoyo y atención al paciente en regiones como Bolivar, Mérida, Carabobo, y otras. Motivando al Estado para la capacitación de nuestros galenos en el trasplante y la necesidad de un boletín informativo para pacientes y médicos.

Misión: La FUNDAVHIP está conformada por un conjunto de personas cuya misión principal es divulgar información sobre HP, orientar sobre que hacer y donde acudir una vez realizado el diagnóstico, canalizar los recursos para la investigación sobre HP y ayudar a personas con escasos recursos para los procedimientos diagnósticos y la adquisición de medicamentos y tratamientos.

La clave del éxito es ser la única Fundación en Venezuela de apoyo a los hipertensos pulmonares del país.

\section{LAS SOCIEDADES DE HIPERTENSIÓN PULMONAR PARA PACIENTES EN LATINOAMÉRICA}

En la actualidad, no existen estadísticas del número de pacientes con hipertensión pulmonar en Latinoamérica. Resulta preocupante que en algunos países no se cuenta con médicos especializados en el tema, esto condiciona un retraso en el diagnóstico, dificultad para conseguir los medicamentos y problemas para obtener información de esta entidad en el idioma correspondiente. Este entorno, hace que los pacientes y familiares en el momento del diagnóstico, perciban este con incertidumbre y vulnerabilidad. A esto se suma, no contar con políticas publicas dirigidas a esta enfermedad crónica.

En la última década se observa un crecimiento de las Organizaciones no gubernamentales (ONGs) en algunos países para apoyar a los pacientes Latinos, estos organismos tienen como objetivo común apoyar a la investigación, a la medicina clínica y la educación, con el propósito que el paciente con hipertensión pulmonar tenga una mejor calidad de vida y pronóstico con los tratamientos adecuados.

En el año 2005 se originó en la Florida, USA, la Sociedad Latina de Hipertensión Pulmonar (SLHP) una or- ganización sin fines de lucro. La SLHP es un paso subsecuente que comenzó en Venezuela en el año 2001, como una organización no lucrativa denominada Fundación Amigos de Migdalia Denis (FUNDAMIDE). La SLHP está trabajando para cambiar el panorama sombrío que afecta a todas aquellas personas con hipertensión pulmonar, creando conciencia y adoptando medidas preventivas. Actualmente, esta ONG tiene un importante potencial en términos de factores como flexibilidad, agilidad, compromiso, cercanía a la comunidad y disposición de ayuda. Es la única organización de esta naturaleza para gente latina, dentro y fuera de los EE.UU.

Casi paralelamente son fundadas, la Asociación Brasileña de Amigos y Familiares de Pacientes con Hipertensión Pulmonar, la Asociación Mexicana de Hipertensión pulmonar, la Fundación de Apoyo para la Hipertensión Pulmonar del Sureste en México, la Fundación Venezolana de Hipertensión Pulmonar, la Fundación Colombiana de Hipertensión Pulmonar, la Asociación Civil de Hipertensión Pulmonar Argentina, la Asociación Peruana de Hipertensión Pulmonar, y en forma más reciente han surgido Grupos de Apoyo a pacientes en Puerto Rico, en el Sur de la Florida y en Santo Domingo en Republica Dominicana.

El impulso y participación social que se viene observando en algunos países Latinos en los que se están integrando las ONG, con los científicos, las instituciones publicas y privadas; se debe tomar como ejemplo creando conciencia para el desarrollo y expansión a otros países, permitiendo un cambio en el panorama en que viven los pacientes con hipertensión pulmonar en toda Latinoamérica.

\section{BIBLIOGRAFIA}

1. Chin KM and Rubin LJ: Pulmonary arterial hypertension. J Am Coll Cardiol. 2008; 51(16):1527-38.

2. McLaughlin VV, Archer SL, Badesch DB, Barst RJ, Farber HW, Lindner JR et al: ACCF/AHA 2009 expert consensus document on pulmonary hypertension a report of the American College of Cardiology Foundation Task Force on Expert Consensus Documents and the American Heart Association developed in collaboration with the American College of Chest Physicians; American Thoracic Society, Inc.; and the Pulmonary Hypertension Association. J Am Coll Cardiol. 2009;53(17):1573-619

3. Galiè N, Torbicki A, Barst R, Dartevelle P, Haworth S, Higenbottam $T$ et al: Guidelines on diagnosis and treatment of pulmonary arterial hypertension. The Task Force on Diagnosis and Treatment of Pulmonary Arterial Hypertension of the European Society of Cardiology. Eur Heart J. 2004;25(24):2243-78.

4. Badesch D, Champion H, Gomez MA, Hoeper M, Loyd J, Manes $A$ et al: Diagnosis and assessment of pulmonary arterial hypertension. J Am Coll Cardiol. 2009;54(1 Suppl):S55-66

5. Sitbon O, Humbert M, Jaïs X, loos V, Hamid AM, Provencher S et al: Long-term response to calcium channel blockers in idiopathic pulmonary arterial hypertension. Circulation. 2005;111(23):310511 
6. Simonneau G, Robbins IM, Beghetti M, Channick RN, Delcroix M, Denton $\mathrm{CP}$ et al: Updated clinical classification of pulmonary hypertension. J Am Coll Cardiol 2009; 54(1 Suppl): S43-5.

7. Trow TK, McArdle JR: Diagnosis of pulmonary arterial hypertension. Clin Chest Med 2007; 28(1): 59-73, viii.

8. Barst RJ, McGoon M, Torbicki A, Sitbon O, Krowka MJ, Olschewski $\mathrm{H}$, et al. Diagnosis and differential assessment of pulmonary arterial hypertension. J Am Coll Cardiol. 2004;43(12 Suppl S):40S-47S.

9. Schannwell C, Steiner S, Strauer B. Diagnostics in pulmonary hypertension. J Physiol Pharmacol. 2007; 58 (Suppl 5(Pt 2): 591-602.

10. Hegewald MJ, Markewitz B, Elliott CG: Pulmonary hypertension: clinical manifestations, classification and diagnosis. Int J Clin Pract Suppl 2007; (156): 5-14.

11. Tuder RM, Abman SH, Braun T, Capron F, Stevens T, Thistlethwaite PA et al: Pulmonary circulation: development and pathology. J Am Coll Cardiol 2009; 54(1 Suppl): S3-9.
12. Rubin LJ; American College of Chest Physicians: Diagnosis and management of pulmonary arterial hypertension: ACCP Evidencebased clinical practice guidelines. Chest 2004;126(1 Suppl):7S$10 \mathrm{~S}$.

13. Boutet K, Montani D, Jaïs X, Yaïci A, Sitbon O, Simonneau G et al: Therapeutic advances in pulmonary arterial hypertension. Ther Adv Respir Dis 2008; 2(4): 249-65.

14. Galiè N, Palazzini M, Leci E, Manes A: Current Therapeutic Approaches to Pulmonary Arterial Hypertension. Rev Esp Cardiol 2010; 63(6): 708-724.

15. Provencher $S$, Jais $X$, Yaici A, Sitbon $O$, Humbert M, Simonneau G: Clinical challenges in pulmonary hypertension: Roger $S$. Mitchell lecture. Chest 2005; 128(6 Suppl): 622S-628S.

16. Condliffe R, Kiely DG, Peacock AJ, Corris PA, Gibbs JS, Vrapi F et al: Connective tissue disease-associated pulmonary arterial hypertension in the modern treatment era. Am J Respir Crit Care Med 2009; 179(2): 151-7. 